\title{
CONTROLE AVANÇADO DE PRESSÃO APLICADO A UM REATOR DE LEITO FLUIDIZADO DE UMA INDÚSTRIA PETROQUÍMICA
}

\author{
E. RECH, L. A. FARINA, M. S. CADORE e G. A. NEUMANN \\ Braskem S.A. \\ eloisa.rech@braskem.com
}

\begin{abstract}
RESUMO - Este artigo apresenta uma estratégia de controle avançado multivariável aplicada a um reator que opera em leito fluidizado de uma unidade de produção de copolímeros de uma indústria petroquímica. Uma maior produtividade de copolímeros é consequência, dentre outras variáveis, de uma pressão maior no reator. Foi desenvolvido um MPC (Model Predictive Controller, Controlador Preditivo baseado em Modelo) com o objetivo de controlar e maximizar a pressão deste reator, sob condições de processo restritas e seguras. O controle de pressão era realizado pelo operador atuando nas malhas de nível do reator e de vazão de recirculação de gás na sucção do compressor; após a modificação, manipula-se automaticamente o nível do reator de forma preditiva. Reduziu-se a variabilidade da pressão do reator, a qual possibilita uma operação mais próxima do limite máximo de pressão. São estimados benefícios em produtividade para alguns produtos e melhoria na troca térmica no resfriador de gases do reator.
\end{abstract}

\section{INTRODUÇÃO}

A Braskem é hoje a maior produtora de resinas termoplásticas nas Américas, maior produtora mundial de biopolímeros com o polietileno verde e maior produtora de polipropileno nos Estados Unidos, integrando operações de primeira e segunda gerações da petroquímica no Brasil, obtendo vantagens competitivas, como escala de produção e eficiência operacional.

Dentro do complexo produtivo da Braskem, existem 14 plantas de polipropileno, resina que apresenta características como baixo custo, excelente balanço de propriedades físicas, possibilidade de alta transparência, resistência ao impacto em baixas temperaturas e baixa densidade. Para melhorar a propriedade de resistência do material, algumas plantas da Braskem produzem copolímero de propeno e eteno, realizando a reação de produção da resina em dois tipos de reatores, o que é o caso do processo de produção foco deste estudo.

A planta Bulk1 da PP1, em Triunfo-RS, utiliza o processo de produção Spheripol ${ }^{\circledR}$, com dois reatores em fase líquida e um reator em fase gás, como descrito na seção seguinte. $\mathrm{O}$ foco deste trabalho é apresentar o desenvolvimento e os resultados de um projeto de aumento de carga da planta em uma condição específica onde o reator fase gás é o gargalo de produção.

Um problema em plantas de polimerização é a grande demanda por troca térmica. Para a 
planta em estudo, verificou-se um gargalo ao aumento de produção referente exatamente a este problema, no reator fase gás. Uma forma de melhorar a troca térmica do reator fase gás é aumentar a pressão de operação. Este objetivo já era buscado pelo operador de painel, atuando sobre algumas variáveis de processo. A ideia do projeto foi automatizar estas ações do operador, reduzindo a variabilidade da pressão e maximizando a mesma, sob condições seguras. Por se tratar de um processo multivariável e com restrições, a escolha da solução técnica de controle automático de processos apontou para o uso de Controle Preditivo Multivariável.

\section{DESCRIÇÃO DO PROCESSO}

O polipropileno é produzido nas plantas Spheripol, conforme fluxograma simplificado apresentado na Figura 1. O polímero é formado dentro dos reatores loop em fase líquida de propeno, em uma reação de polimerização em massa (Bulk polimerization). Para produção de copolímero, uma segunda etapa de reação, agora em fase gás com leito fluidizado, é conduzida inserindo-se no meio reacional o comonômero eteno.

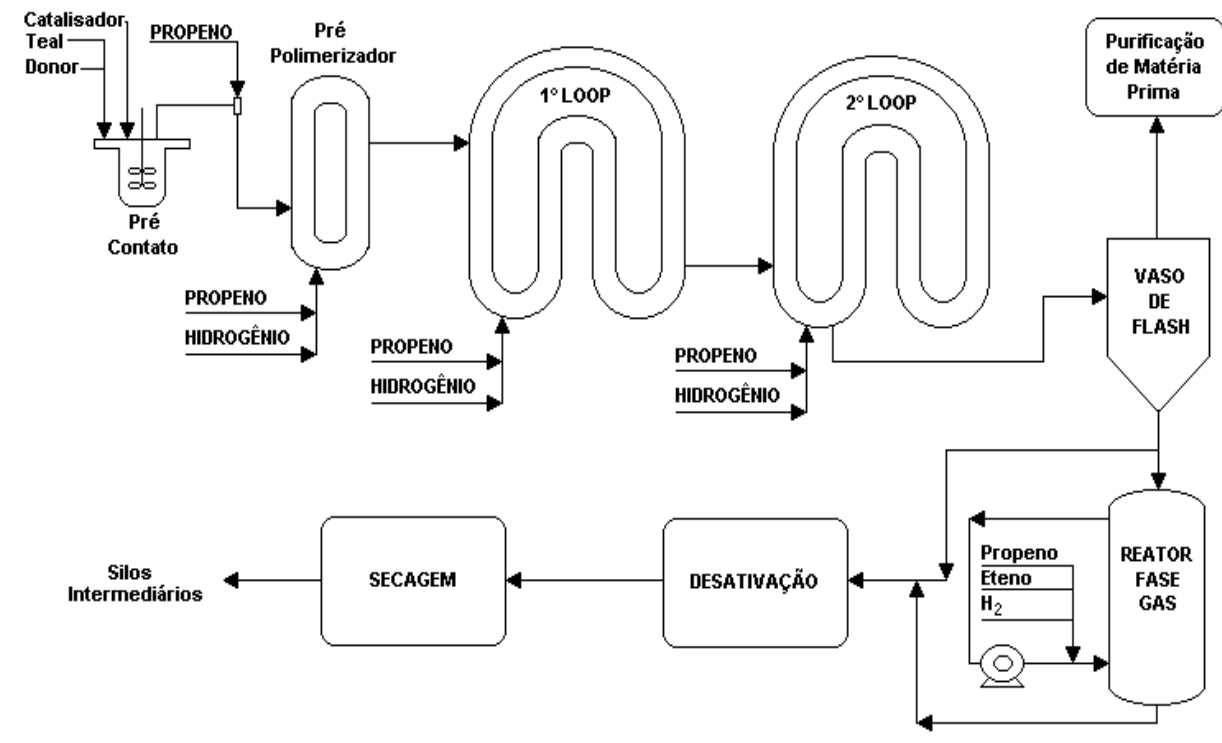

Figura 1 - Fluxograma simplificado do Processo Spheripol.

As reações de polimerização são exotérmicas e, no sistema reacional, o eteno é mais reativo que o propeno, assim, em campanhas onde se trabalha com altos teores de eteno incorporado à resina surgem limitações de troca térmica no sistema do reator fase gás. A produtividade fica limitada pela transferência de calor.

\section{CONTROLE DE PRESSÃO}

Para controlar a pressão de operação do reator fase gás, o operador atuava manualmente na válvula de recirculação de gás para o sistema (HV02), na sucção do compressor, e no setpoint do controle de nível do leito fluidizado no reator (LIC01). Um aumento na abertura da válvula de recirculação implica em um aumento na pressão, pois reduz a perda de carga; já um aumento no nível do leito fluidizado acarreta em uma redução da pressão, pois aumenta a área de reação, convertendo mais gás em resina. 
Para garantir a segurança do sistema, em condições de pressão excessivamente elevada, um controle automático atuava aliviando para purga parte do inventário de reagentes (PV02). Uma vez que esta purga é bastante prejudicial dos pontos de vista econômico e operacional, a tendência da operação era manter a pressão do sistema em um ponto confortável, abaixo do ponto de atuação do controle automático de purga. Em oposição a esta tendência, sabe-se que aumentando a pressão de operação, em especial na condição de copolímero de alto teor de eteno, tem-se um considerável ganho na troca térmica (no trocador E01), que é um gargalo para o aumento de carga da planta.

A figura 2 ilustra esquematicamente o reator fase gás (RFG), o sistema de troca térmica no trocador (E01) e reciclo com o compressor (C01). As principais variáveis envolvidas com o controle de pressão são apresentadas na figura.

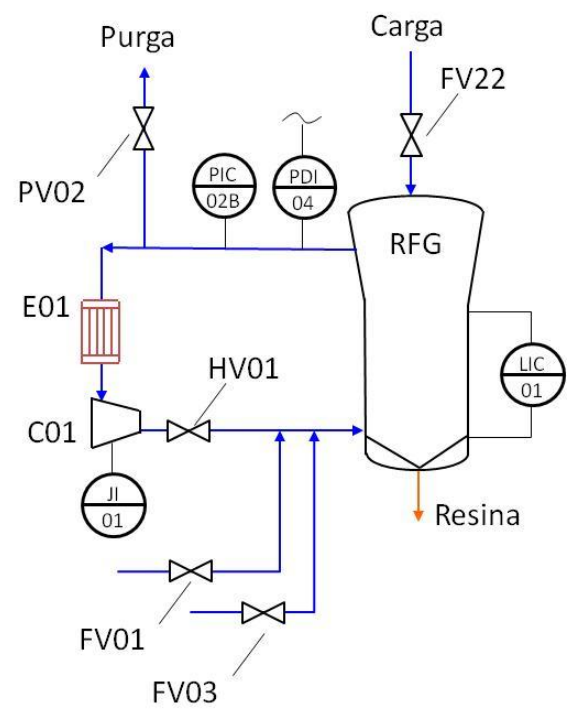

Figura 2 - Fluxograma do reator fase gás, com sistema de troca térmica e reciclo.

A oportunidade de redução da variabilidade da pressão com a automação dos procedimentos do operador já era prevista no projeto da planta, porém, historicamente, nunca teve bons resultados. Avaliando-se o problema, verificou-se a multivariabilidade presente no processo, o que justifica os problemas históricos no uso de um controle simples para o fim desejado e indica o uso de estratégias de controle avançado.

Utilizou-se um modelo empírico para o desenvolvimento de um MPC (Model Predictive Controller, Controlador Preditivo baseado em Modelo) pela representatividade dos dados de processo disponíveis. Badgwell e Qin (2000) descreveram o termo MPC como uma classe de algoritmos computacionais que controlam o comportamento futuro de uma planta através da utilização de um modelo de processo explícito. A identificação do modelo é descrita na seção seguinte.

\subsection{Identificação do modelo empírico}

O processo de identificação de um modelo empírico costuma ser conduzido a partir de testes em que o sistema em estudo é submetido a perturbações nas variáveis de entrada. Segundo Bemporad e Morari (1999), o objetivo é obter parâmetros de modelo que minimizem o somatório 
da diferença entre os valores de saída da planta e os valores preditos pelo modelo, ou seja, que capturem o comportamento do sistema em um horizonte de futuro. Deste modo, mudanças nas variáveis controladas (CVs) ou restrições (CCVs) são preditas devido a alterações nas variáveis manipuladas (MVs) ou distúrbios (DVs).

As principais variáveis que afetam a pressão do sistema são as vazões de propeno e eteno frescos para o reator fase gás (FIC03 e FIC01), e a vazão de propeno de reciclo do sistema de separação dos reatores fase líquida para o reator fase gás (FIC22). Estas variáveis definem as composições do meio reacional, definindo o tipo de resina produzida e estando definidas pela receita de cada produto. Tem-se ainda as já citadas variáveis independentes, utilizadas para o ajuste da pressão: LIC01 e HV02.

A variável controlada é a pressão (PIC02B), mas o controle afeta também outras variáveis de processo que devem ser monitoradas e controladas por faixa: a potência do compressor (JI01) não pode exceder os limites seguros de operação da máquina e o diferencial de pressão no sistema a montante do reator fase gás (PDI04) não pode comprometer o escoamento de gás para o reator.

Com o levantamento das variáveis mais importantes, foi montada a matriz de controle apresentada na Tabela 1, que mostra o comportamento relativo esperado para as variáveis de saída com base no conhecimento de processo.

Tabela 1 - Matriz de controle do sistema.

\begin{tabular}{|c|c|c|c|}
\hline CV/CCVs & PIC02B & JI01 & PDI04 \\
\hline LVs/DVs & & & $\uparrow \uparrow \uparrow$ \\
\hline HV02.OUT & $\uparrow \uparrow$ & $\downarrow \downarrow \downarrow$ & $\downarrow \downarrow$ \\
\hline FIC03.PV & $\uparrow$ & $\uparrow \uparrow \uparrow$ & $\downarrow$ \\
\hline FIC22.PV & $\uparrow$ & $\uparrow$ & $\downarrow$ \\
\hline FIC01.PV & $\uparrow$ & - & - \\
\hline
\end{tabular}

De acordo com Morari (1998), convencionalmente, os modelos utilizados nas aplicações de MPC são identificados através de uma série de testes na planta. Entretanto, devido ao cenário competitivo do setor, que possui baixa margem de erro permitido, evitou-se a inserção de distúrbios ao sistema. Assim, o procedimento utilizado limitou-se à seleção de períodos representativos resultantes de uma análise sistemática de dados históricos. Estes dados foram obtidos através de um banco de dados de processos e, após tratamento, exportados para o software Pavilion $8^{\circledR}$. Ainda neste contexto, verificou-se que o controle deveria atender a condições de processso de teor de eteno alto, médio e baixo. Como o reator fase gás opera, predominantemente, com teor médio de eteno, os parâmetros do modelo foram obtidos para esta condição e validados para as demais condições.

Selecionaram-se períodos de variação das variáveis manipuladas e dos distúrbios de processo em separado, cuja resposta da variável controlada fosse nítida e cujos sinais dos ganhos estivessem de acordo com a matriz de controle apresentada na Tabela 1.

Os dados históricos utilizados e os modelo preditos (P) para as variáveis de saída 
(variáveis controlada e restrições) em condição de teor médio de eteno estão representados na Figura 2. O período corresponde a, aproximadamente, vinte dias, com frequência de um ponto por minuto. Devido a sigilo de informações industriais, os valores reais das variáveis estão suprimidos; de qualquer forma, o importante para os modelos de controle são os ganhos relativos e as dinâmicas, bem representados na figura.

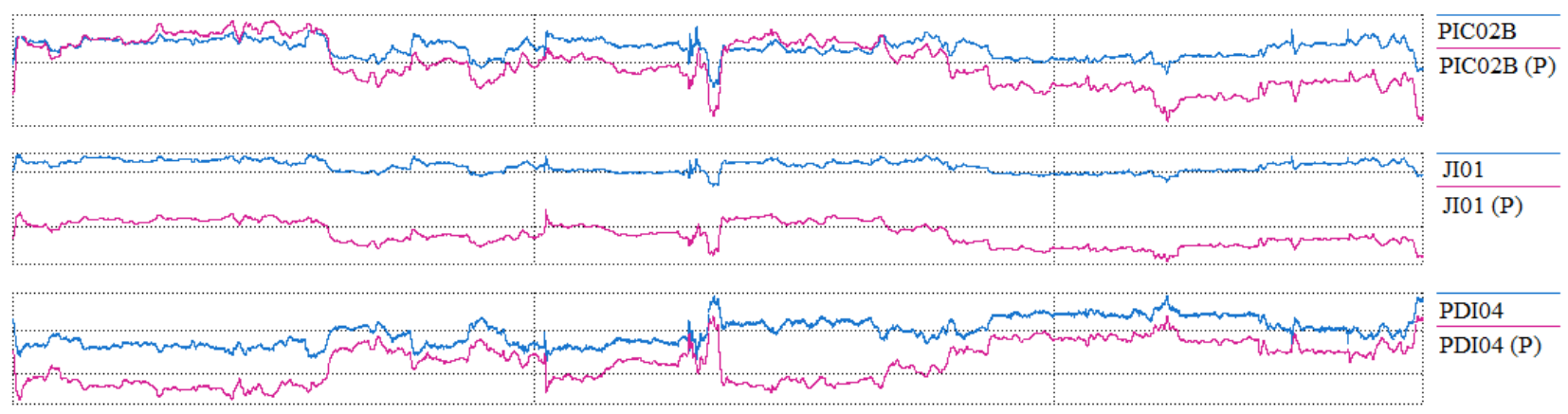

Figura 2 - Simulação do modelo gerado em Pavilion ${ }^{\circledR}$ para as variáveis de saída em condição de teor médio de eteno.

Uma vez que o modelo matemático é baseado em dados empíricos para o cálculo de seu s pontos ótimos de operação, deve-se verificar sua validade para situações e períodos de processo diferentes. Esta validação, correspondente a quatro meses de dados históricos, está representada na Figura 3 e não faz distinção a teores de eteno.

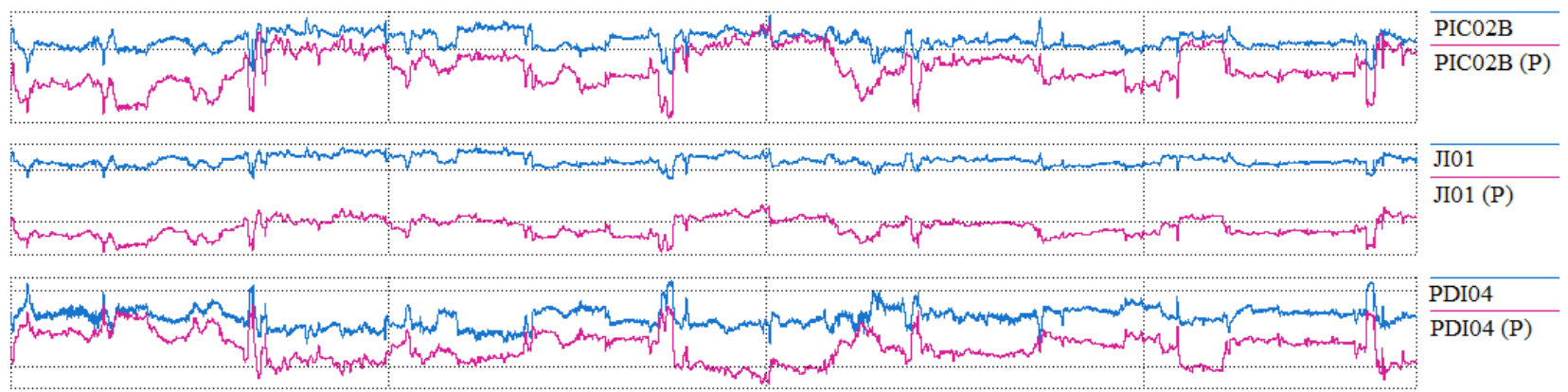

Figura 3 - Validação do modelo gerado em Pavilion® para as variáveis de saída sem distinção de teor de eteno.

Analisando-se as Figuras 2 e 3, conclui-se que os modelos simulados apresentam desempenho satisfatório, representando bem o comportamento real do processo. É relevante citar que o distanciamento entre as tendências preditas e reais apresentadas nas figuras não interferem no desempenho do controlador preditivo, já que o modelo se corrige através do acréscimo da diferença entre o valor real e o predito. Distúrbios de processo não previstos na matriz de controle também são minimizados através desta técnica de correção de modelo.

Por fim, implementou-se um analisador virtual com base no modelo desenvolvido, a fim de disponibilizar a inferência da tendência da pressão do reator fase gás para as etapas iniciais do comissionamento. 


\subsection{Comissionamento}

Qualquer modificação na Braskem deve passar por um processo de Gestão de Mudança. Para o comissionamento do controle multivariável de pressão não foi diferente. Anteriormente à implementação, uma equipe multidisciplinar envolvendo engenharias de automação, produção e processo, com apoio da equipe de segurança de processos, avaliou os potenciais riscos envolvidos com a mudança.

Após aprovada a mudança, foi realizado o teste offline do controlador, que consistiu em avaliar as predições do modelo do controlador para as modificações realizadas pelo operador, sem ação automática do controlador sobre o processo. Com o próprio modelo foi possível realizar simulações da ação que o controlador tomaria quando em operação online, estas ações eram efetivadas pelo operador e as respostas do processo comparadas às previstas pelo simulador.

Quando confirmada a assertividade do modelo de predição e das ações propostas pelo controlador, passou-se à etapa de atuação efetiva do controlador preditivo sobre o processo. Por um período de cerca dois meses o controlador foi ligado apenas em horário administrativo, com acompanhamento da engenharia de automação. Durante o período, a equipe de operação se habituou aos procedimentos envolvidos com o novo controlador, entendendo também os objetivos e ganhos com o sistema.

Finalmente, foi realizado treinamento formal da equipe de operação, com entrega do documento de referência rápida para uso do controle avançado, e então o controlador foi considerado implementado.

A partir da entrega do controlador, iniciou-se o levantamento de capturas com o sistema e a sustentação de valor, que busca garantir a continuidade de uso.

\section{RESULTADOS E BENEFÍCIOS}

Com o uso do controlador multivariável, foi possível verificar uma redução na variabilidade da pressão do reator, sendo possível operar com a válvula de água para o trocador de calor mais fechada, o que permite um aumento de carga.

A Figura 4 mostra os efeitos de redução na variabilidade da pressão, aumento na pressão média (em azul) e consequente fechamento da válvula de água de resfriamento (em vermelho). Nota-se que o controle leva a variabilidade da pressão para o nível (setpoint em rosa). 


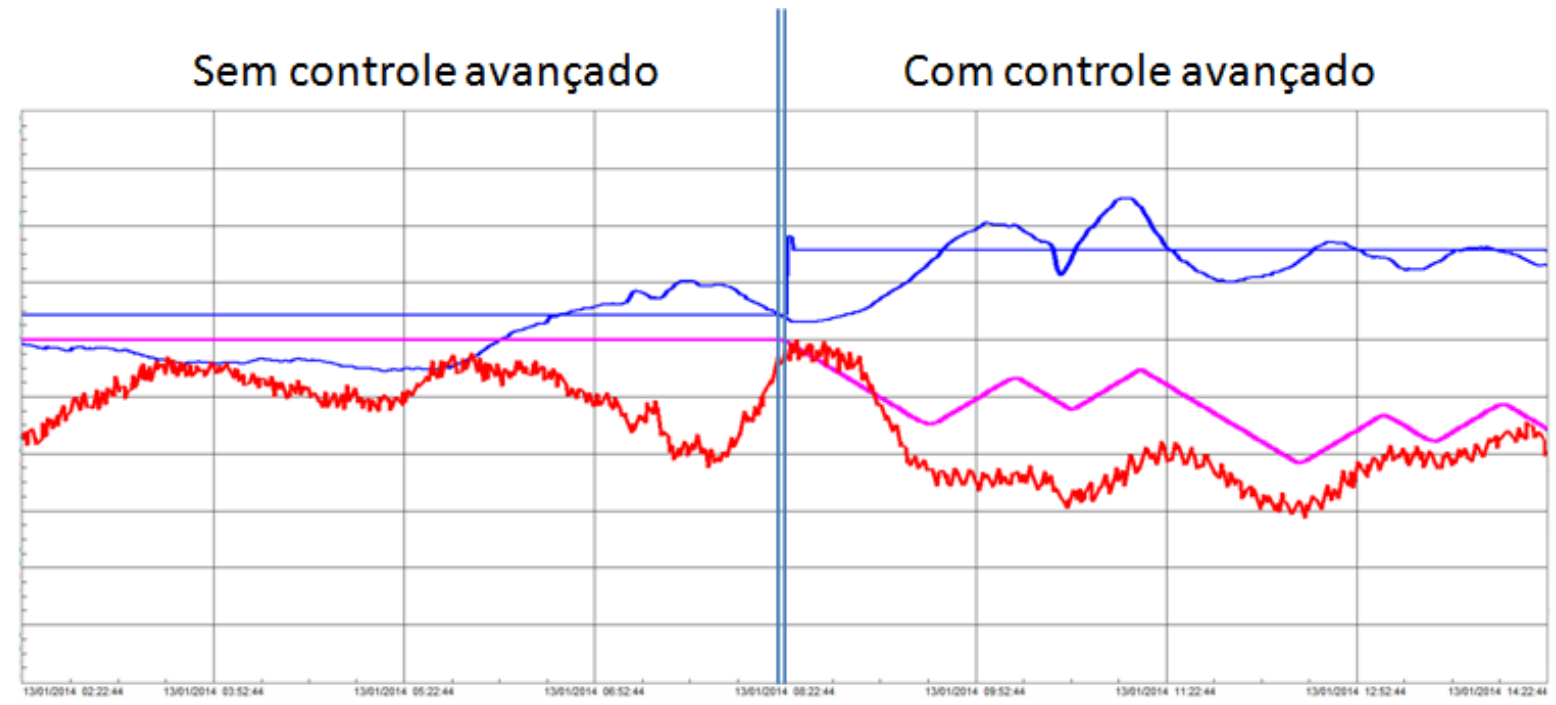

Figura 4 - Principais resultados alcançados com o uso do controlador multivariável.

O levantamento preliminar de ganhos com o controle avançado foi realizado a partir do balanço de energia do reator fase gás. Existe uma correlação direta da produção de resina com o calor trocado no E01, este pode ser determinado pela Equação 1, da troca térmica a partir do calor específico a pressão constante.

$$
\mathrm{Q}=\mathrm{m} \cdot \mathrm{C}_{\mathrm{p}} \cdot \Delta \mathrm{T}
$$

Avaliando o aumento de pressão média no reator, possível a partir da redução de variabilidade proporcionada pelo controle multivariável, e seu efeito na válvula de controle de água, que influencia no $\Delta \mathrm{T}$ expresso na Equação 1, se obtém um aumento estimado em 3,4\% no ganho de troca térmica. Este mesmo ganho, convertido em carga nas campanhas de alto teor de eteno incorporado, representa um potencial de aumento de cerca de 1.600 toneladas de resina por ano.

\section{CONCLUSÃO}

Embora pouco utilizado para controle de pressão, uma vez que normalmente é uma variável de dinâmica rápida, neste caso um controlador preditivo multivariável se mostrou bastante adequado para um bom controle de pressão no reator fase gás da planta PP1 Bulk1.

Com o novo controle se conseguiu uma redução na variabilidade na variável, permitindo uma operação com maior pressão média, oferecendo um potencial aumento de produção, principalmente para condições de copolímero com alto teor de eteno.

Está em estudo o uso de outra variável que influencia no controle da pressão de reação, e ainda segue sob ação manual da operação, a vazão de recirculação de gás no sistema, assim o controlador passaria a possuir duas variáveis manipuladas, permitindo estratégias de otimização do processo. 


\section{REFERÊNCIAS}

BEMPORAD, A.; MORARI, M. Robust model predictive control: a survey. Robustness in identification and control, p. 1, 1999.

MORARI, M; LEE, J. H. Model predictive control: past, present and future. Comp. and Chem. Eng., v. 23, p. 675, 1998.

QIN, S. J.; BADGWELL, T. A. An overview of nonlinear model predictive control applications.

Nonlinear Predictive Control, p. 369-393, 2000. 